\title{
Pubovaginal Sling with a Low-Cost Polypropylene Mesh
}

\author{
Fransber R. Rodrigues, Romulo Maroccolo Filho, Roberto R. Maroccolo, Lucio C. Paiva, \\ Fernando A. Diaz, Eduardo C. Ribeiro
}

Service of Urology, Brasilia University Hospital, Brasilia, DF, Brazil

\begin{abstract}
Objective: The aim of this study was to present the results of pubovaginal sling with a low-cost polypropylene mesh in the treatment of stress urinary incontinence.

Materials and Methods: 118 women diagnosed with stress urinary incontinence (SUI) due to urethral hypermobility or intrinsic sphincteric deficiency, treated with pubovaginal sling (PVS) with a low-cost polypropylene mesh confectioned by the surgeon, were analyzed. All patients had a basic evaluation that included a medical history, physical examination, stress tests and urodynamic investigation.

Results: The average follow up was of 42 months. Urethral hypermobility was observed in $67 \%$ of the cases. The process was carried out on an outpatient basis on 67 patients. Intra-operative complications included 4 vesical injuries, treated with catheterization for 3 days. There were 13 early complications, of which 8 were urinary retentions treated with vesical drainage for 1 to 3 weeks and 3 vaginal extrusions of the mesh treated with covering of the sections with mucous membrane. There was a need for 6 urethrolysis in patients who presented irritative and postoperative obstructive symptoms; $81.3 \%$ of the patients were considered cured, while $9.3 \%$ had significant improvement. Three initially unsuccessful cases required sling reconfiguration. All cases were eventually cured.

Conclusion: The construction of a pubovaginal sling using a low-cost polypropylene mesh is a safe and effective technique for the relief of SUI. It should be considered an alternative, especially for patients in public health systems with low financial resources.
\end{abstract}

Key words: urinary incontinence, stress; prostheses and implants; suburethral slings; polypropylene

Int Braz J Urol. 2007; 33: 690-94

\section{INTRODUCTION}

Suburethral slings have become the preferred technique for the treatment of stress urinary incontinence (SUI) (1). The Integral Theory and concept of a medium tension free urethral sling, surgical refinements and new materials allowed these procedures to be carried out in a non invasive manner, with low morbidity rates and satisfactory results (2-4).

Various materials were used in the slings, from autogenous tissues to synthetic materials, and the choices were based upon well-defined criteria, in which the cost was one of the most important factors for the selection of materials, mainly in countries with limited public health resources (5). 
Studies confirm that the choice of a tension free polypropylene mesh allow high success rates and the TVT ${ }^{3}$ simplified the SUI therapy, becoming one of most common options for the treatment of this disease $(3,6)$. Thus, the industry has offered different kits to make the slings, but most of the time the costs are prohibitive for public health systems with few financial resources.

The objective of this study is to analyze the results of pubovaginal sling (PVS) using a low-cost polypropylene mesh for the treatment of stress urinary incontinence (SUI).

\section{MATERIALS AND METHODS}

A retrospective study of 118 women with SUI treated with PS using a low cost polypropylene mesh was conducted from April 2002 to April 2006. The preoperative evaluation consisted of the medical history, urogynecological examination, stress tests and a urodynamic assessment cystometry, measure of the leak point pressure and flux-pressure study. The etiology of SUI was considered urethral hypermobility when the leak point pressure under stress (VLPP) was greater than $60 \mathrm{cmH}_{2} \mathrm{O}$, and intrinsic sphincteric deficiency (ISD) when the pressure was below that value.

The follow up was carried out with consultations in the first month after the treatment and every 4 months, in which stress tests and clinical histories were obtained. Urodynamic studies were indicated in patients with persistence of SUI, irritative and/or moderate obstructive symptoms, or urinary retention 30 days after the surgical procedure.

Patients who related satisfaction and did not express urinary loss after stress tests were considered cured. Patients in use of pads for precaution, due to minimal urinary loss, but satisfied with the results were considered patients with significant recovery. The other cases were classified as failures.

\section{Surgical Technique}

All the procedures were carried out under spinal anesthesia, except 1 patient who underwent concomitant laparoscopic surgery. Patients received

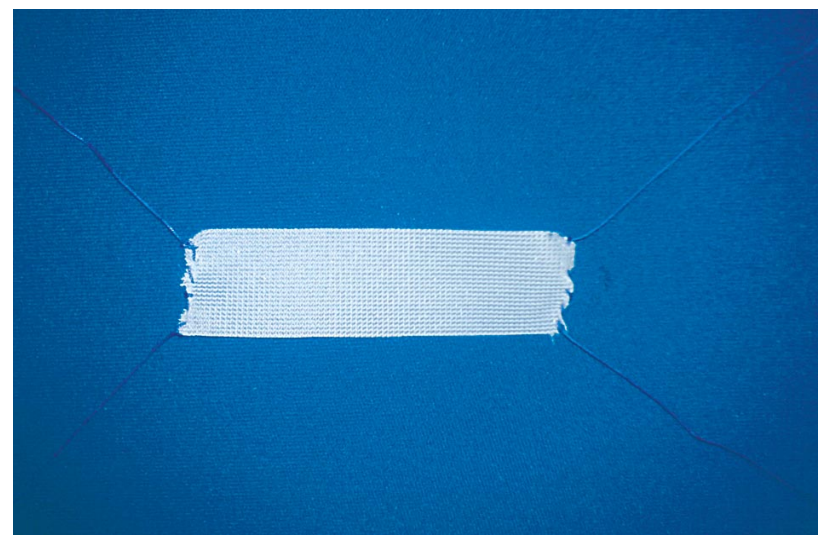

Figure 1 - Polypropylene mesh with poygalactyne-0 thread fixed to the extremities, confectioned by the surgeon.

first generation cephalosporin as a prophylactic antibiotic. The surgical technique adopted was similar to the technique described by Almeida and Raz (6), using a polypropylene mesh of $10 \times 1.5 \mathrm{~cm}$, with polygalactyne- 0 threads fixed at each extremity of the mesh, confectioned by the surgeon (Figure-1).

A longitudinal incision of $2 \mathrm{~cm}$ was made on the anterior vaginal wall, $1 \mathrm{~cm}$ from the urethral meatus, and dissection of the periurethral spaces was done. The endopelvic fascia was opened on each side, with access to the retropubic space. A hypogastric incision of $2 \mathrm{~cm}$ was made on the upper border of the pubic bone, allowing the passage of the long tongs to the retropubic space, bringing them out through the vaginal incision at each side of the urethra. The threads at the extremities of the mesh were held by the tongs and pulled till the abdominal incision, where they were tied after confirmation of the absence of vesical injuries by cystoscopy, in such a manner that the mesh stays below the urethra, free of tension.

\section{RESULTS}

The average age was 52 years (29-77 years). From the 118 patients, 2 had been treated previously with Burch colposuspension and 23 received surgical indications after failure of perineal physiotherapy. Eighty patients were diagnosed with urethral hypermobility $(67 \%)$, from which 4 presented detrusor hyperactivity, and 38 were diagnosed with ISD 
(33\%). The stress test was positive in all cases. Fifty six women (48\%) used pads daily, with an average of $1.7 \mathrm{pad} /$ day. The medium VLPP was $84 \mathrm{cmH}_{2} \mathrm{O}$. Fiftytwo patients (44\%) presented irritative symptoms before the surgery.

Procedures associated to PVS were carried out in 17 cases, described in Table-1. The average operative time was 49 minutes, including other concomitant procedures. The surgery was carried out on an outpatient basis on 67 patients (56\%), with an average operative time of 28 minutes. Four vesical injuries $(3.3 \%)$ were observed during the procedures and were treated with a vesical tube for 3 days. No cases of substantial hemorrhage were observed.

Post-operative complications are described in Table-2. All cases of acute urinary retention were resolved with vesical tubes for 1-3 weeks. The 3 cases of vaginal mesh extrusion $(2.5 \%)$ were treated with coverage by the vaginal mucosa, with success in 2 patients. The third patient presented recurrent ero-

Table 1 - Procedures associated with the pubovaginal sling.

\begin{tabular}{lc}
\hline Procedures & $\begin{array}{c}\text { Number } \\
\text { of Cases }\end{array}$ \\
\hline Posterior perineoplasty & 10 \\
Vaginal hysterectomy + perineoplasty & 2 \\
Vaginal hysterectomy & 1 \\
Excision of Gardner cyst & 1 \\
Abdominal hysterectomy & 1 \\
Paraurethral cyst correction & 1 \\
Laparoscopic treatment of renal cyst & 1 \\
\hline
\end{tabular}

sion, requiring removal of the mesh segment, but persisting continent.

Average post-operative follow up was 42 months (14-61 months). After the procedure, 5 patients presented irritative urinary symptoms "de novo" (4.2\%), without detrusor hyperactivity on postoperative urodynamics. All patients with preoperative detrusor hyperactivity continued with irritative symptoms, however without SUI. From the 52 patients with preoperative irritative symptoms, 40 (76.9\%) reported improvement of symptoms. There was necessity for urethrolysis in 6 patients $(5 \%)$ who continued with significant irritative symptoms and a suspicious infravesical obstruction on urodynamics. Three patients from this group continued continent, while the others underwent a new PVS using the initial technique, and presented incontinence resolution.

Ninety six patients $(81.3 \%)$ were considered cured, and 11 patients $(9.3 \%)$ presented significant improvement of incontinence. From the 11 women who did not benefit from the treatment, 3 underwent a new PVS, achieving continence. The others are being followed up by the perineal physiotherapy staff.

\section{COMMENTS}

The Integral Theory postulates that female urinary continence occurs due to the closing of the medium portion of the urethra, which depends on the integrity of pubourethral ligaments and the suburethral support given by the anterior wall of the vagina. Similar to $\mathrm{TVT}^{\circledR}$, the technique discussed here is based on this theory, positioning the sling in the medial third of the urethra, free of tension. The principal difference

Table 2 - Postoperative complications and treatments.

\begin{tabular}{lcc}
\hline Complication & Number of Cases & Treatment \\
\hline Pubic hematoma & 1 & Conservative \\
Acute vaginitis & 1 & Antibiotics \\
Vaginal erosion of mesh & 3 & Covering of the mesh with vaginal mucosa \\
Acute urinary retention & 8 cases removal (1 case) & Bladder drainage (1 to 3 weeks) \\
\hline
\end{tabular}


lies in the cost of the material used, since in this study the same propylene mesh was used, however it was prepared by the surgeon, instead of the specific commercialized material for this procedure. The propylene mesh and polygalactyne thread approximately costs U\$ 15.00 .

The sling made of autogenous tissue, like the abdominal rectus fascia or fascia lata, has shown good long-term results. However, the high morbidity rates associated to the procedure pose a disadvantage (79). The use of cadaverous fascia would eliminate some inconveniences and, in short term, produce similar results to that observed with autogenous slings (10); however, further studies have not confirmed these initial good results (11). This scenario led to the development of synthetic materials for slings, especially polypropylene, which is very durable, has low indices of rejection and is easily available.

The main intraoperative complications of PVS were vesical perforation and hemorrhage. Bousted (4), in a metanalysis, observed $6.9 \%$ of vesical perforations in 160.000 patients treated with TVT $^{\circledR}$. Tamoussino et al. (12) verified vesical injuries in $2.7 \%$ of 2.795 patients from the Austrian series. According to data from Meshia et al. (13), bleeding was reported in $15 \%$ of the cases. In this series, low incidence of intraoperative complications was observed, including 4 vesical injuries (3.3\%), and there were no significant cases of hemorrhage.

Postoperative complications included 8 cases of urinary retention (6.7\%), and vaginal erosion of the mesh in 3 patients $(2.5 \%)$; there were also isolated cases of pubic hematoma and acute vaginitis. A variable incidence of urinary retention after the TVT ${ }^{\circledR}$ was reported $(2.3 \%-43 \%)$, in which an increase is observed when associated to other pelvic procedures $(14,15)$. The vaginal erosion of the mesh was observed in approximately $1 \%$ of the cases. Patients who presented this complication were treated with simple procedures, with good results.

Resolution of preoperative irritable symptoms was observed in $76.9 \%$ of the women, and $4.2 \%$ presented "de novo" urinary urgency, without detrusor hyperactivity. The incidence of this symptom is not consistent in literature, being reported in $1 \%$ to $35 \%$ of patients $(16,17)$. In this study, obstructive and/or irrita- tive symptoms persisted in 6 patients (5\%), and all underwent urethrolysis, with improvement in all cases.

Bousted analyzed the results from 16 studies about TVT ${ }^{\circledR}$, with a minimum follow up of 12 months, observing objective cure rates of $80 \%$ to $96 \%$, and $5 \%$ to $17 \%$ of significant improvement (4). In the present study, the cure rate $(81.3 \%)$ and significant improvement $(9.3 \%)$ are similar to those obtained by TVT $^{\circledR}$, even in patients diagnosed with ISD, whose results tend to be worse. Rezapour et al. (18) obtained cure in $74 \%$ and an important improvement in $12 \%$ of the patients with ISD that underwent sling with TVT ${ }^{\circledR}$.

\section{CONCLUSION}

Complications and cure rates of the pubovaginal sling (PVS) using a low cost polypropylene mesh can be compared to those of TVT ${ }^{6}$ for the treatment of SUI, with an advantage of lower costs. This procedure should be considered as an alternative to PVS with commercial kits, mainly for patients of public health systems with few financial resources.

\section{CONFLICT OF INTEREST}

None declared.

\section{REFERENCES}

1. Niknejad K, Plzak LS 3rd, Staskin DR, Loughlin KR: Autologous and synthetic urethral slings for female incontinence. Urol Clin North Am. 2002; 29: 597-611.

2. Petros PE, Ulmsten UI: An integral theory and its method for the diagnosis and management of female urinary incontinence. Scand J Urol Nephrol Suppl. 1993; 153: 1-93.

3. Leach GE, Dmochowski RR, Appell RA, Blaivas JG, Hadley HR, Luber KM, et al.: Female Stress Urinary Incontinence Clinical Guidelines Panel summary report on surgical management of female stress urinary incontinence. The American Urological Association. J Urol. 1997; 158: 875-80.

4. Bousted GB: The tension-free vaginal tape jfor treating female stress urinary incontinence. BJU int. 2002; 89: 687-9. 
5. Palma PC: Which sling for which patient? Int Urogynecol J. 2004; 15: 369-2.

6. Rodriguez LV, Raz S: Prospective analysis of patients treated with a distal urethral polypropylene sling for symptoms of stress urinary incontinence: surgical outcome and satisfaction determined by patient driven questionnaires. J Urol. 2003; 170: 857-63; discussion 863.

7. Bemelmans BL, Chapple CR: Are slings now the gold standard treatment for the management of female urinary stress incontinence and if so which technique? Curr Opin Urol. 2003; 13: 301-7.

8. Cross CA, Cespedes RD, McGuire EJ: Our experience with pubovaginal slings in patients with stress urinary incontinence. J Urol. 1998; 159: 1195-8.

9. Elliott DS, Boone TB: Is fascia lata allograft material trustworthy for pubovaginal sling repair? Urology. 2000;56: 772-6.

10. Singla AK: The use of cadaveric fascia lata in the treatment of stress urinary incontinence in women. BJU Int. 2000; 85: 264-9.

11. O'Reilly KJ, Govier FE: Intermediate term failure of pubovaginal slings using cadaveric fascia lata: a case series. J Urol. 2002; 167: 1356-8.

12. Tamussino K, Hanzal E, Kolle D, Ralph G, Riss P, Austrian Urogynecology Working Group: The Austrian tension-free vaginal tape registry. Int Urogynecol J Pelvic Floor Dysfunct. 2001; 12 (Suppl 2): S28-9.
13. Meschia M, Pifarotti P, Bernasconi F, Guercio E, Maffiolini M, Magatti F, et al.: Tension-Free vaginal tape: analysis of outcomes and complications in 404 stress incontinent women. Int Urogynecol J Pelvic Floor Dysfunct. 2001; 12 (Suppl 2): S24-27.

14. Kuuva N, Nilsson CG: A nationwide analysis of complications associated with the tension-free vaginal tape (TVT) procedure. Acta Obstet Gynecol Scand. 2002; 81:72-7.

15. Partoll LM: Efficacy of tension-free vaginal tape with other pelvic reconstructive surgery. Am J Obstet Gynecol. 2002; 186: 1292-5; discussion 1295-8.

16. Debodinance P, Delporte P, Engrand JB, Boulogne M: Tension-free vaginal tape (TVT) in the treatment of urinary stress incontinence: 3 years experience involving 256 operations. Eur J Obstet Gynecol Reprod Biol. 2002; 105: 49-58.

17. Fynes M, Murrey C, Carey M, Rosamilia A, Dwyer P: Prognostic factors for continence outcome following tension-free vaginal tape. An observational study. Int Urogynecol J Pelvic Floor Dysfunct. 2000; 11 (suppl 1): S33.

18. Rezapour M, Falconer C, Ulmsten U: Tension-Free vaginal tape (TVT) in stress incontinent women with intrinsic sphincter deficiency (ISD) - a long-term followup. Int Urogynecol J Pelvic Floor Dysfunct. 2001; 12 (Suppl 2): S12-14. 